Proceedings of the 2010 Winter Simulation Conference

B. Johansson, S. Jain, J. Montoya-Torres, J. Hugan, and E. Yücesan, eds.

\title{
MACHINE QUALIFICATION MANAGEMENT FOR A SEMICONDUCTOR BACK-END FACILITY
}

\author{
Mengying Fu \\ Moeed Haghnevis \\ Ronald Askin \\ John Fowler \\ Muhong Zhang \\ School of Computing, Informatics and Decision Systems Engineering \\ Arizona State University \\ Tempe, AZ 85287, USA
}

\begin{abstract}
In order to process a product in a semiconductor back-end facility, a machine needs to be qualified first by having a product-specific software program installed on it. Then a tool set must be available and attached on the machine while it is processing the product. In general not all machines are qualified to process all products due to the high machine qualification cost and tool set availability. However, the machine qualification decision is very important because it affects capacity allocation in the facility and subsequently affects daily production scheduling. To balance the tradeoff between the machine qualification costs and backorder costs, a product-machine qualification optimization model is proposed in this paper.
\end{abstract}

\section{INTRODUCTION}

The semiconductor manufacturing process consists of two main parts: the front-end process and the back-end process. The front-end process, also known as wafer fabrication, typically has a small number of products and very complex reentrant product flow. In contrast, the back-end process, also known as assembly and test, typically has hundreds or thousands of different products and relatively linear product flow. The research presented in this paper focuses on the back-end process.

In a semiconductor back-end facility, each machine has to be configured for each of the products it will process in the future. This configuration (machine qualification) process includes installing and testing a software program for each product on the machine. Due to the wide product mix (i.e. thousands of products), if all machines were to be qualified for all products, the machine qualification process could take a really long time, thus incurring a high nonnegligible machine qualification cost. Meanwhile, not all machines are even able to be qualified for all products. Because of short product life cycles and fast development of new products in the semiconductor industry, machines need to be updated with new products. As a result, machines that perform the same operation could belong to different machine types/generations, with each type/generation only being able to be qualified for a subset of products. In addition, the product-machine qualification decision affects the capacity planning decision and subsequently the daily production scheduling in the future. Poor product-machine qualification decisions could cause shortages by not qualifying enough machines for a given product or machine utilization imbalance by qualifying too many products on a small subset of machines. In this paper, a mixed integer linear programming model (MILP) is proposed to optimize product-machine qualification while considering production scheduling in a medium term planning horizon (e.g. a couple of weeks). As the last part of the semiconductor manufacturing system, on time delivery of customer orders is generally the most important goal in the back-end process. Hence the objective of the MILP is set to minimize the weighted machine qualification costs and backorder costs.

The remainder of the paper is organized as follows. Section 2 is a literature review about machine qualification optimization. In Section 3, a mixed integer linear programming model (MILP) is presented to optimize product-machine qualification at a stage in the semiconductor back-end process. This is followed by Section 4, in which extensions of the MILP model are discussed. Finally, conclusions and future research directions are provided in Section 5. 


\section{LITERATURE REVIEW}

Product-machine or operation-machine qualification is a very common feature in the modern semiconductor manufacturing process. A few papers consider this feature in their scheduling models (Hurink et al. 1994, Jurisch 1995, Brucker et al. 1997, Mati and Xie 2004, Wu et al. 2006, Wu et al. 2008), but none of them proposes any change or optimization on the current machine qualification. There are also some other papers that utilize short-term machine dedication to schedule the production (Campbell 1992, Bourland and Carl 1994). An operation-machine qualification management system is proposed by Johnzén et al. (2008) for a semiconductor front-end facility, and four flexibility measures are developed for each operation-machine qualification. Impact of different operation-machine qualifications, with different scores according to each of the four flexibility measures, on scheduling is showed through simulation. Aubry et al. (2008) present a mixed integer linear programming model (MILP) for the product-machine qualification optimization of parallel multi-purpose machines. The objective is to minimize machine configuration costs while still obtaining a load-balanced capacity allocation. The MILP formulation is proved to be strongly NP-hard but could be relaxed to a transportation problem under some assumptions. Rossi (2010) provide a robustness measure for the multi-purpose machine configuration model based on Aubry et al. (2008). Maximal disturbance of the demand that would change the optimal configuration is used as the robustness measure. Ignizio (2009) proposes a binary optimization model for operation-machine qualification at the photolithography machines in a wafer fabrication factory. The objective is to obtain a load-balanced schedule at minimal machine qualification costs. The cycle time in the factory is shown to be decreased using the binary optimization model compared to qualifications developed by heuristic or "educated guess" means. In somewhat related work, Drexl and Mundschenk (2008) propose an integer programming model for long-term employee staffing based on qualification profiles. The objective is to accomplish all tasks with minimal total employment costs. Employee scheduling could be another application area of the methodologies developed for the machine qualification management in the factory.

However, none of these papers above considers multi-stage manufacturing systems or production scheduling in the machine qualification optimization model. In this research, a mixed integer linear programming model (MILP) is proposed to optimize product-machine qualification in a multi-stage manufacturing system while considering detailed scheduling in a medium term planning horizon (e.g. several weeks). The MILP minimizes the total machine qualification costs and backorder costs.

\section{MACHINE QUALIFICATION OPTIMIZATION MODEL (MQO)}

A mixed integer linear programming model (called MQO) is proposed to optimize product-machine qualification in a semiconductor back-end facility. The facility has multiple stages with parallel machines at each stage. Products are processed in lots with a fixed numbers of units in each lot. Setup times are sequence-dependent and not included in the lot processing time. However, setup times are not modeled explicitly in MQO. Instead, the setup times are considered by decreasing the machine capacity by a certain percentage (defined as $1-A$ in the MQO). Product-machine qualification is considered in the model, and thus only qualified machines can process a given product at a given step. Initial product-machine qualifications could be provided though an existing qualification matrix. If the demand is not completely satisfied with the initial product-machine qualifications, additional machines will be qualified while balancing backorder costs and qualification costs. The planning horizon is divided into small time buckets to model the material handling/movement in the system. Meanwhile, the production quantity of each product on each machine will be scheduled for each time bucket.

\section{Notation:}

$P$ : number of products, with index $p$

$N$ : number of stages, with index $n$

$M[n]$ : number of unrelated machines at stage $n$

$T$ : number of time periods, with index $t$

$C$ : capacity of a machine in each time period $\left(C_{n, m}\right.$ if it is machine dependent)

$A$ : available percentage of the machine capacity for production in each time period after setup reservation

$B_{p, 0}$ : initial back order quantity of product family $p$

$I_{p, n, 0}$ : initial inventory of product $p$ at (after) stage $n$

$b_{p}$ : back order cost of product $p$

$d_{p, t}:$ demand quantity of product $p$ at the end of time period $t$

$t_{p, n, m}$ : unit processing time of product $p$ on machine $m$ at stage $n$

$q[p, n, m]: 1$ if machine $m$ at stage $n$ is qualified for product $p, 0$ otherwise

$c_{p, n, m}$ : cost of qualifying machine $m$ at stage $n$ for product $p$

Decision Variables:

$X_{p, n, m, t}$ : continuous variable, production quantity for product $p$ in time period $t$ on machine $m$ at stage $n$

$I_{p, n, t}$ : continuous variable, inventory quantity of product $p$ at the end of time period $t$ after stage $n$

$B_{p, t}$ : continuous variable, back order quantity of the product $p$ at the end of time period $t$ 
$Q_{p, n, m}$ : binary variable, 1 if machine $m$ at stage $n$ is recommended to be qualified for product $p, 0$ otherwise

$$
\begin{array}{ll}
\text { (MQO) } & \\
\text { min } & b_{p} B_{p, t}{ }_{p, t}{ }_{p, n, m_{q[p, n, m]=0}} c_{p, n, m} Q_{p, n, m} \\
\text { s.t. } & I_{p, n, t-1}+{ }_{m} X_{p, n, m, t}-{ }_{m} X_{p, n+1, m, t}=I_{p, n, t}, \text { for all } p, n<N, t \\
& I_{p, N, t-1}-B_{p, t-1}+{ }_{m} X_{p, N, m, t}-d_{p, t}=I_{p, N, t}-B_{p, t}, \text { for all } p, t \\
& X_{p, n+1, m, t} \leq I_{p, n, t-1}, \text { for all } p, n<N, t
\end{array}
$$

The objective (1) is to minimize the total backorder and machine qualification costs. Constraints (2) are the inventory balance constraints for every product at every stage, except the last stage, in each time period. They indicate that the inventory quantity at the end of current time period must equal to the previous inventory plus production minus consumption at the next stage. Constraints (3) are the inventory balance constraints for every product at the last stage in each time period. They are similar to constraints (2) except that the consumption at the next stage is replaced by demand. Backorders are allowed but incur cumulative backorder costs as shown in the objective expression (1). Constraints (4) are the material availability constraints, which state that the production quantity at stage $n$ in period $t$ must be less than the inventory quantity at stage $n-1$ in period $t-1$. Constraints (5) are the capacity constraints for every machine in each time period, which state that the total production time must be less than the available machine capacity after setup time reservation. Constraints (6) are the machine qualification constraints, which allow the production quantities to be positive if the machine is qualified for the product. Constraints (7) and (8) are the positive and binary constraints for the decision variables.

The model could be easily extended to include different process routes for different products and material handling time between stages by slightly modifying the subscripts. For example, instead of $X_{p, n+1, m, t}, X_{p, n+2, m, t}$ should be used in constraints (2) and (4) if product $p$ skips stage $n+1$. The material handling time for product $p$ between stage $n$ and stage $n+1$ needs to be added on to the subscript $t$ of all $X_{p, n+1, m, t}$ 's in constraints (2) and (4).

\section{MACHINE QUALIFICATION MANAGEMENT SYSTEM}

A machine qualification management system with a graphical user interface was developed with Excel macro based on the above machine qualification optimization model. The architecture of the system is shown in Figure 1. First, data from the factory are transformed using the input generator into formatted text files. There are three categories of data. The first category consists of static factory data, which includes physical production system information (e.g. number of stages, number of parallel machines at each stage), process information (e.g. lot processing time of each product on each machine), product information (e.g. number of products, priority for each product), and a matrix of existing machine qualifications. The static data do not change frequently. The second category consists of the dynamic demand information for the planning period. Those data could change from week to week and can be changed by the user to do a what-if analysis. The third category consists of user preferences. First users will be asked to provide the preference between each pair of machines, which is determined by the distance between the machines. For example, machines close to each other are preferred to be qualified for the same product so that the material handling time is shorter. Then users need to input the preference between each pair of machine and product, which may be determined by the lot processing time for a product on a machine. Usually faster machines are more preferred.

The formatted text files are then read in by the qualification feasibility checker. The qualification feasibility checker utilizes the MQO model but sets all additional machine qualification variables $Q_{p, n, m}$ 's to 0 . The output of the qualification feasibility checker consists of machine utilizations and backorder quantities in each time period. If there are backorders, users will have two options (manual or automatic) for qualifying additional machines to decrease the backorder quantities. In the manual qualification optimization mode, the utilization of each machine in each time period will be presented to the user, who could then improve the machine qualification based on expert knowledge. As the automatic qualification optimization mode is chosen, the MQO model will be used to identify additional machines to be qualified while balancing the machine qualification and backorder costs. Machine utilization and backorder reports 
are generated from the MQO model.

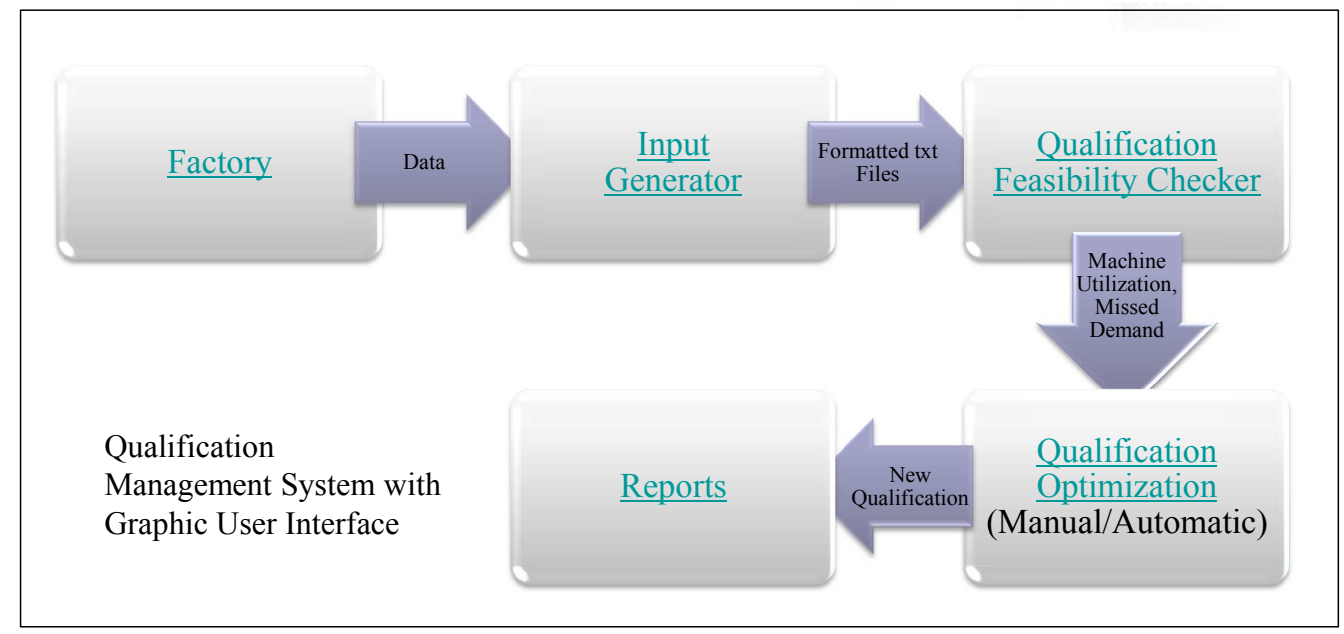

Figure 1: Architecture of the machine qualification management system.

All the above functions are integrated in a graphical user interface (GUI) with a report generator. The GUI has three main screens: run, report, and analyze. In the run screen, users set parameters for the MQO model, such as the available percentage of the machine capacity $A$ and a percentage parameter used to change the demand quantity for what-if analysis. Furthermore, users can input and modify machine-machine preferences and product-machine preferences. The report generator in the GUI generates a weekly report, which is the report screen, showing the capacity of each machine used on each product and the backorder quantity of each product for each week in the planning horizon. Figure 2 shows a sample report for 2 weeks in the report screen. Take the week 1 report for example, the available machine capacity for production (Machine Availability $A$ ) and machine capacity spent on production (Effective Utilization $U$ ) are listed for each machine, with the percentage of machine capacity spent on each product on the right side of the table. On the bottom of the table are the demand (QTG, which a factory term short for quantity to go), production quantity (Completed), and end-of-week shortage (Accumulative Miss) for each product. In the analyze screen, users can choose whether to manually qualify machines for products with backorders or run the automatic qualification optimization algorithm to obtain qualification recommendations that balance the qualification and backorder costs.

\subsection{AN EXAMPLE}

An example is presented here, with 2 products and 3 stages. Product 1 has a higher priority of 5 and product 2 has a lower priority of 1 . There are 8,10, and 9 machines at the first, second, and third stages, respectively. Parallel machines have different processing times for the same product, as shown in Table 1. P $x$ represents product $x$, and $\mathrm{S} y \_$means the $z$ th machine at stage $y$. If the lot processing time is 0 , e.g. for product 1 on machine 1 at stage 3 , the machine can not be qualified for the product. The initial product machine qualification matrix is shown in Table 2, with 1 meaning the machine is qualified for a given product and 0 otherwise. The planning period is limited to 3 weeks, with the weekly demands for product 1 as 0 lot, 38 lots, and 0 lot, and for product 2 as 38 lots, 87 lots, and 88 lots.

Table 1: Lot processing time (hr) of each product on each machine.

\begin{tabular}{|c|c|c|c|c|c|c|c|c|c|c|}
\hline & $\begin{array}{l}\text { S1_1 } \\
\end{array}$ & S1_2 & S1_3 & S1_4 & S1_5 & S1_6 & S1_7 & S1_8 & & \\
\hline P1 & 2.05 & 2.05 & 2.03 & 2.03 & 2.05 & 2.05 & 2.03 & 2.05 & & \\
\hline \multirow[t]{2}{*}{$\mathrm{P} 2$} & 1.73 & 1.75 & 1.49 & 1.49 & 1.49 & 1.49 & 1.49 & 1.49 & & \\
\hline & S2_1 & $\mathrm{S} 2 \_2$ & S2_3 & S2_4 & S2_5 & S2_6 & S2_7 & S2_8 & S2_9 & S2_10 \\
\hline P1 & 2.07 & 2.07 & 2.07 & 2.07 & 2.07 & 2.07 & 2.07 & 2.07 & 2.07 & 2.07 \\
\hline \multirow[t]{2}{*}{$\mathrm{P} 2$} & 1.55 & 1.55 & 1.55 & 1.55 & 1.55 & 1.55 & 1.55 & 1.55 & 1.55 & 1.55 \\
\hline & $\begin{array}{l}\text { S3_1 } \\
\text {. }\end{array}$ & S3_2 & S3_3 & S3_4 & S3_5 & S3_6 & S3_7 & S3_8 & S3_9 & \\
\hline P1 & 0.00 & 2.05 & 2.05 & 2.05 & 2.05 & 2.05 & 2.05 & 2.07 & 2.05 & \\
\hline P2 & 0.00 & 2.37 & 2.37 & 2.37 & 2.37 & 2.37 & 2.37 & 2.37 & 2.37 & \\
\hline
\end{tabular}



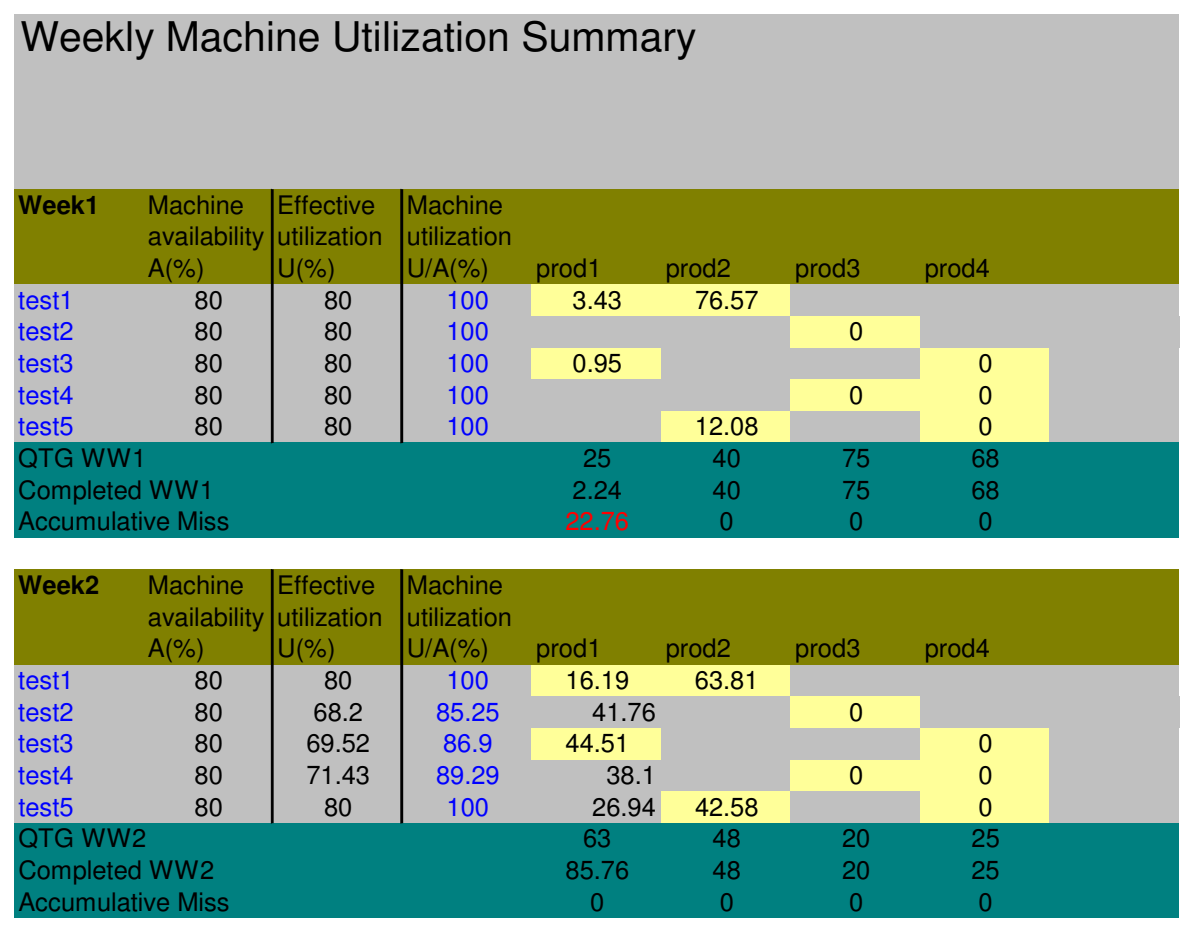

Figure 2: Report of the machine qualification management system.

The results of qualification feasibility checker and qualification optimization with two different $A$ values are shown in Table 3. It should be noted that a negative number for shortage means inventory at the end of the week. Comparing the shortages and total costs between current qualification and optimized qualification, we can conclude that the qualification optimization eliminated the shortage with minimal machine qualification cost for the example. In addition, the value of $A$ affects the feasibility checker and qualification optimization results. Therefore, choosing a proper $A$ value is very important for the machine qualification management system. In the next Section 4.2, a potential solution for obtaining a proper $A$ value is presented.

\subsection{INTEGRATION WITH A SCHEDULING SYSTEM}

The production scheduling considered in the MQO model is simplified compared to the real shop floor scheduling, as it does not consider the sequence-dependent setups or the non-preemptive machine scheduling rules. The available percentage of the machine capacity for production $A$ is generally inaccurate. If $A$ is set to be larger than the actual value, it might cause shortages in the real production. On the other hand, if $A$ is set to be smaller than the actual value, it could cause unnecessary machine qualification cost. As a result, the machine qualification management system

Table 2: Initial product-machine qualification.

\begin{tabular}{|l|rrrrrrrrrr|}
\hline & S1_1 & S1_2 & S1_3 & S1_4 & S1_5 & S1_6 & S1_7 & S1_8 & & \\
\hline P1 & 1 & 1 & 0 & 1 & 1 & 1 & 0 & 1 & & \\
P2 & 1 & 1 & 0 & 0 & 0 & 0 & 0 & 0 & & \\
\hline & S2_1 & S2_2 & S2_3 & S2_4 & S2_5 & S2_6 & S2_7 & S2_8 & S2_9 & S2_10 \\
\hline P1 & 0 & 0 & 0 & 1 & 0 & 1 & 0 & 1 & 1 & 0 \\
P2 & 1 & 1 & 0 & 0 & 0 & 0 & 0 & 0 & 0 & 0 \\
\hline & S3_1 & S3_2 & S3_3 & S3_4 & S3_5 & S3_6 & S3_7 & S3_8 & S3_9 & \\
\hline P1 & 0 & 0 & 0 & 1 & 0 & 1 & 0 & 1 & 0 & \\
P2 & 0 & 0 & 0 & 0 & 0 & 0 & 0 & 1 & 1 & \\
\hline
\end{tabular}


Table 3: Computational result.

\begin{tabular}{|c|c|c|c|c|c|c|c|c|}
\hline & \multicolumn{4}{|c|}{$A=80 \%$} & \multicolumn{4}{|c|}{$A=90 \%$} \\
\hline & \multicolumn{2}{|c|}{$\begin{array}{c}\text { Current } \\
\text { Qualification }\end{array}$} & \multicolumn{2}{|c|}{$\begin{array}{c}\text { Optimized } \\
\text { Qualification }\end{array}$} & \multicolumn{2}{|c|}{$\begin{array}{c}\text { Current } \\
\text { Qualification }\end{array}$} & \multicolumn{2}{|c|}{$\begin{array}{c}\text { Optimized } \\
\text { Qualification }\end{array}$} \\
\hline & P1 & P2 & $\mathrm{P} 1$ & P2 & P1 & P2 & P1 & P2 \\
\hline Week1 Shortage & -16.41 & -18.81 & -27.87 & -31.55 & -8.79 & -25.91 & -28.03 & -30.04 \\
\hline Week2 Shortage & 0 & 11.38 & 0 & -17.1 & 0 & -2.82 & 0 & -16.08 \\
\hline Week3 Shortage & 0 & 42.57 & 0 & 0 & 0 & 21.27 & 0 & 0 \\
\hline Backorder Cost & \multicolumn{2}{|c|}{78.1} & \multicolumn{2}{|c|}{0} & \multicolumn{2}{|c|}{17.3} & \multicolumn{2}{|c|}{0} \\
\hline Qualification Cost & \multicolumn{2}{|c|}{0} & \multicolumn{2}{|c|}{7.8} & \multicolumn{2}{|c|}{0} & \multicolumn{2}{|c|}{7.8} \\
\hline Total Costs & \multicolumn{2}{|c|}{78.1} & \multicolumn{2}{|c|}{7.8} & \multicolumn{2}{|c|}{17.3} & \multicolumn{2}{|c|}{7.8} \\
\hline
\end{tabular}

should be integrated with a scheduling system, in which the suggested machine qualification could be verified and the parameter $A$ could be adjusted.

\section{CONCLUSION}

In this paper, a mixed integer linear programming model (MILP) is proposed to optimize product-machine qualifications for a semiconductor back-end facility. Production scheduling in a medium term planning horizon is considered and setup times are modeled indirectly. Based on the MILP model, a Excel-based machine qualification management system with a graphical user interface (GUI) is developed to be used in the factory.

In future research, the MILP model and the management system with GUI will be tested with real data and in a real factory. An interesting topic will be adding stochastic factors in the optimization of the machine qualification, such as the demand uncertainty and random machine breakdowns.

\section{REFERENCES}

Aubry, A., A. Rossi, M. Espinouse, and M. Jacomino. 2008. Minimizing setup costs for parallel multi-purpose machines under load-balancing constraint. European Journal of Operational Research 187 (3): 1115-1125.

Bourland, K., and L. Carl. 1994. Parallel-machine scheduling with fractional operator requirements. IIE Transactions 26 (5): 56-65.

Brucker, P., B. Jurisch, and A. Krämer. 1997. Complexity of scheduling problems with multi-purpose machines. Annals of Operations Research 70:57-73.

Campbell, G. 1992. Using short-term dedication for scheduling multiple products on parallel machines. Production and Operations Management 1 (3): 295-307.

Drexl, A., and M. Mundschenk. 2008. Long-term staffing based on qualification profiles. Mathematical Methods of Operations Research 68 (1): 21-47.

Hurink, J., B. Jurisch, and M. Thole. 1994. Tabu search for the job-shop scheduling problem with multi-purpose machines. OR Spectrum 15 (4): 205-215.

Ignizio, J. 2009. Cycle time reduction via machine-to-operation qualification. International Journal of Production Research 47 (24): 6899-6906.

Johnzén, C., P. Vialletelle, S. Dauzère-Pérès, C. Yugma, and A. Derreumaux. 2008. Impact of qualification management on scheduling in semiconductor manufacturing. In Proceedings of the 40th Conference on Winter Simulation, ed. S. Mason, R. Hill, L. Monch, T. Jefferson, and J. Fowler, 2059-2066: Piscataway, New Jersey: Institute of Electrical and Electronics Engineers.

Jurisch, B. 1995. Lower bounds for the job-shop scheduling problem on multi-purpose machines* 1. Discrete Applied Mathematics 58 (2): 145-156.

Mati, Y., and X. Xie. 2004. The complexity of two-job shop problems with multi-purpose unrelated machines. European Journal of Operational Research 152 (1): 159-169.

Rossi, A. 2010. A robustness measure of the configuration of multi-purpose machines. International journal of production research 48 (3-4): 1013-1033.

Wu, M., Y. Huang, Y. Chang, and K. Yang. 2006. Dispatching in semiconductor fabs with machine-dedication features. The International Journal of Advanced Manufacturing Technology 28 (9): 978-984.

Wu, M., H. Jiang Jr, and W. Chang. 2008. Scheduling a hybrid MTO/MTS semiconductor fab with machine-dedication features. International Journal of Production Economics 112 (1): 416-426. 


\section{AUTHOR BIOGRAPHIES}

MENGYING FU is currently pursuing the doctoral degree in the Industrial Engineering program of the School of Computing, Informatics, and Decision Systems Engineering at Arizona State University, Arizona State, US. Her academic interests include production scheduling and integer optimization. She received the B.S. in Industrial Engineering from Tsinghua University, Beijing, China. Her email address is <Mengying.Fu@asu.edu>.

MOEED HAGHNEVIS is a PhD student of Industrial Engineering in the School of Computing, Informatics, and Decision Systems Engineering at Arizona State University. He received his M.Sc. and B.S. in Industrial and System Engineering from University of Tehran and Amirkabir University of Technology, Iran, respectively. His interests include engineering complex systems, computer simulation, and project management. He has published a book in project management and several journal and conference papers. Before starting his $\mathrm{PhD}$, he served as an Adjunct Instructor at Iran University of Science and Technology and Amirkabir University of Technology.

RONALD ASKIN is a Professor of Industrial Engineering and Director of the School of Computing, Informatics, and Decision Systems Engineering at Arizona State University. He received his PhD from Georgia Institute of Technology and has 30 years of experience in the development, teaching and application of methods for production systems analysis. $\mathrm{He}$ is a Fellow of IIE and has published extensively. His list of awards includes a National Science Foundation Presidential Young Investigator Award, the Shingo Prize for Excellence in Manufacturing Research, IIE Joint Publishers Book of the Year Award, and the IIE Transactions Development and Applications Award.

JOHN W. FOWLER is the Avnet Professor of Supply Networks and Program Chair of Industrial Engineering in the School of Computing, Informatics, and Decision Systems Engineering at Arizona State University (ASU). His research interests include modeling, analysis, and control of manufacturing and service systems. His research has been supported by the National Science Foundation, Semiconductor Research Corp., International SEMATECH, Asyst, IBM, Intel, Motorola, Infineon Technologies, ST Microelectronics, and the Mayo Clinic. Dr. Fowler is an author on over 75 journal publications, 100 conference papers, and 10 book chapters. He is the founding editor of the new journal IIE Transactions on Healthcare Systems Engineering. He is also an Area Editor for SIMULATION: Transactions of the Society for Modeling and Simulation International, an Associate Editor for IEEE Transactions on Semiconductor Manufacturing, and on the Editorial Board for the Journal of Simulation. He is a Fellow of the Institute of Industrial Engineers, was recently the INFORMS Vice President for Chapters/Fora, and is on the Winter Simulation Conference Board of Directors.

MUHONG ZHANG is an Assistant Professor of Industrial Engineering in the School of Computing, Informatics, and Decision Systems Engineering at Arizona State University. Dr. Zhang received her B.S. in Applied Mathematics from Beijing University of Chemical Technology, China, in 1999, an M.A. in Operations Research from Chinese Academy of Sciences, in 2002 and a Ph.D. in Industrial Engineering and Operations Research from University of California, at Berkeley, in 2006. Her research interests include integer programming, robust optimization, computational optimization and network problems. Dr. Zhang is a member of the Institute for Operations Research and the Management Sciences (INFORMS). 